\title{
Realistic nuclear Hamiltonian: Ab exitu approach
}

\author{
A. M. Shirokov ${ }^{\mathrm{a}, \mathrm{b}}$, J. P. Vary ${ }^{\mathrm{b}, \mathrm{c}, \mathrm{d}}$, A. I. Mazure ${ }^{\mathrm{e}}$ T. A. Weber ${ }^{\mathrm{b}}$

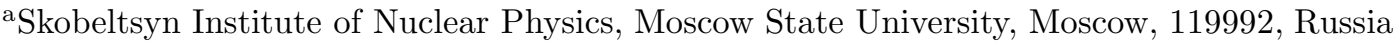 \\ ${ }^{\mathrm{b}}$ Department of Physics and Astronomy, Iowa State University, Ames, Ia 50011-3160, USA \\ ${ }^{\text {c} L a w r e n c e ~ L i v e r m o r e ~ N a t i o n a l ~ L a b o r a t o r y, ~ L-414, ~} 7000$ East Avenue, Livermore, California, 94551, USA \\ ${ }^{\mathrm{d}}$ Stanford Linear Accelerator Center, MS81, 2575 Sand Hill Road, Menlo Park, California, 94025, USA \\ ePacific National University, Tikhookeanskaya 136, Khabarovsk 680035, Russia
}

Fully-microscopic No-core Shell Model (NCSM) calculations of all stable $s$ and $p$ shell nuclei are used to determine a realistic $N N$ interaction, JISP16, describing not only the two-nucleon data but the binding energies and spectra of nuclei with $A \leq 16$ as well. The JISP16 interaction, providing rapid convergence of the NCSM calculations, is obtained in an $a b$ exitu approach by phase-equivalent transformations of the JISP $6 N N$ interaction.

PACS: 21.30.-x, 21.60.Cs, 21.1-.-k, 21.10.Dr

Key words: Inverse scattering $N N$ interaction, No-core Shell Model, light nuclei

To complement the successful but computationally intensive ' $a b$ initio' No-core Shell Model (NCSM) [1, we introduce the ' $a b$ exitu' NCSM. While the former has proven very successful for light nuclei when one includes three-body $(N N N)$ forces [2]3, the computational complexity motivates us to introduce an approach that simultaneously minimizes $N N N$ forces while providing more rapid convergence with a pure nucleon-nucleon $(N N)$ force. We invoke directly an end-goal of nuclear theory (hence the term ' $a b$ exitu'), a successful description of nuclear properties, including the available $N N$ data, to develop a new class of $N N$ potentials that provide accurate descriptions of a broad range of nuclear data.

To achieve this, we form a union of two recent techniques - the $J$-matrix inverse scattering 445]6] and the NCSM [1]. A major ingredient of our approach is the form of the $N N$ interaction (a small matrix in the oscillator basis), which is chosen to provide rapid convergence of manybody observables within the NCSM. Indeed, we show below that results up through $A=16$ obtained directly with the bare interaction (one that accurately describes the $N N$ data) are close to those obtained with the effective interaction and are very useful to establish the confidence region for the binding energy.

Since this is a departure from the more traditional approach, we motivate our development with observations concerning the successful $a b$ initio approaches to light nuclei. Indeed several promising microscopic approaches have been introduced and tested extensively with realistic $N N$ interactions (see [7] and references therein) and with realistic $N N+N N N$ interactions 82 , 3. Progress towards heavier nuclei appears limited only by scientific manpower and by available computers. However, all approaches face the exponentially rising computational complexity inherent in the quantum many-body problem with increasing particle number and novel schemes are needed to minimize the computational burden without sacrificing realism and precision.

The earliest and most successful in reaching nuclei beyond $A=4$ is the Green's-function Monte Carlo (GFMC) approach [8] whose power has been used to determine a sequence of everimproving $N N N$ interactions 89910 , in conjunction with highly precise $N N$ interactions [11] that 
fit a wide selection of low-lying properties of light nuclei up through $A=10$. In addition, the Hamiltonians are tested for their predictions in infinite systems 12. According to our usage of terminology here, the application of GFMC to determine successful $N N N$ interactions is an excellent example of an $a b$ exitu approach.

Now, we ask the question whether it is possible to go even further and search through the residual freedoms of a realistic $N N$ interaction to obtain new $N N$ interactions that satisfy three criteria: (1) retain excellent descriptions of the $N N$ data; (2) provide good fits to light nuclei; and (3) provide improved convergence properties within the NCSM. The challenge to satisfy this triad of conditions is daunting and we are able to provide only an initial demonstration at the present time.

We are supported by the work of Polyzou and Glöckle who demonstrated [13] that a realistic $N N$ interaction is equivalent at the $A=3$ level to a realistic $N N+N N N$ interaction where the new $N N$ force is related to the initial one through a phase-equivalent transformation (PET). The net consequence is that properties of nuclei beyond $A=3$ become dependent on the freedom within the transformations at the $A=3$ level. It seems reasonable then to exploit this freedom and work to minimize the need for the explicit introduction of three and higher body forces. However, we do not surmise that we would be able to eliminate them completely.

We start from the realistic charge-independent $N N$ interaction JISP6 6] that provides an excellent description of the deuteron properties [6] and $N N$ scattering data with $\chi^{2} /$ datum $=1.03$ for the $1992 n p$ data base (2514 data), and 1.05 for the $1999 \mathrm{np}$ data base (3058 data) [14. JISP6 provides also a very good description of the spectra of $p$ shell nuclei, but we find that it overbinds nuclei with $A \geq 10$. To eliminate this deficiency, we exploited PETs to modify the JISP6 in various partial waves. The resulting interaction, hereafter referred to as JISP16 since it is fitted in our $a b$ exitu approach to the spectra and bindings of stable $A \leq 16$ nuclei, can be obtained from the initial ISTP interaction in the same manner as JISP6 in Ref. 6] but with a different set of PET angles. These angles associated with uni- tary transformations (see Refs. 56 for details) mixing the lowest $s$ and $d$ oscillator basis states in the coupled $s d$ waves and the lowest oscillator basis states in the ${ }^{3} p_{2},{ }^{3} p_{1},{ }^{3} p_{0},{ }^{3} d_{2}$ and ${ }^{1} p_{1}$ waves are $\vartheta=-11.0^{\circ},+5^{\circ},-6^{\circ},-10^{\circ},+25^{\circ}$ and $-12^{\circ}$ respectively. The JISP16 matrix elements in the oscillator basis with $\hbar \omega=40 \mathrm{MeV}$ that differ from those of JISP6, are presented in Tables 1 2. The JISP16 predictions for the deuteron rms radius $r_{d}=1.9643 \mathrm{fm}$ and quadrupole moment $Q=0.288585 \mathrm{fm}^{2}$ slightly differ from the JISP6 results since the JISP16 and JISP6 PET angle in the $s d$ coupled waves is slightly different $\left(\vartheta=-11.0^{\circ}\right.$ versus $\left.-11.3^{\circ}\right)$. In this paper, we include all $N N$ partial waves up to $l=4$ and include in Tables 12 the added matrix elements.

Our fitting procedure is one of 'trial-and-error' where we worked with only a few partial waves that we deemed important for these nuclei. We fit only the excitation energies of few lowest ${ }^{6} \mathrm{Li}$ levels and the ${ }^{6} \mathrm{Li}$ and ${ }^{16} \mathrm{O}$ binding energies. To save time, we performed the NCSM calculations in small enough model spaces (up to $10 \hbar \omega$ for ${ }^{6} \mathrm{Li}$ and up to $4 \hbar \omega$ for ${ }^{16} \mathrm{O}$ ). After obtaining a reasonable description of these observables, we checked that the binding energies and spectra of all the rest $s$ and $p$ shell nuclei are well-described in small model spaces. The results presented below are obtained in the $a b$ initio NCSM calculations with the obtained $N N$ interaction, the ab exitu JISP16, in larger model spaces. This description of the binding energies is somewhat worse than the one obtained during the fit in smaller model spaces, however it is still very reasonable. In a future effort, we will perform a thorough search through the space of possible PETs that should further improve the description of nuclear properties while retaining the excellent description of the $N N$ data.

We illustrate our approach with the ${ }^{16} \mathrm{O}$ ground state energy in Fig. 1] The variational principle holds for the bare interaction results; hence the upper bound (UB) for the ground state energy is the minimum of its $\hbar \omega$ dependence in the $6 \hbar \omega$ model space. In the calculations with the effective interaction obtained by the Lee-Suzuki transformation, the quoted result is conventionally associated with the minimum of the $\hbar \omega$ de- 
Table 1

JISP16 non-zero matrix elements in $\hbar \omega=40 \mathrm{MeV}$ units in the uncoupled $N N$ partial waves that differ from the respective JISP6 matrix elements and of the JISP16 matrices in higher partial waves.

\begin{tabular}{|c|c|c|c|}
\hline$V_{n n}^{l}$ & $V_{n, n+1}^{l}=V_{n+1, n}^{l} V_{n, n+2}^{l}=V_{n+2, n}^{l}$ & $V_{n n}^{l}$ & $V_{n, n+1}^{l}=V_{n+1, n}^{l} V_{n, n+2}^{l}=V_{n+2, n}^{l}$ \\
\hline${ }^{1} p_{1}$ partial wave & & \multicolumn{2}{|c|}{${ }^{3} p_{0}$ partial wave } \\
\hline $0 \quad 0.4864373541$ & $-0.2359869829 \quad 0.3117643519$ & $0 \quad 0.1571004930$ & $-0.1425039101 \quad 0.2505691390$ \\
\hline $1-0.1487460250$ & -0.1438603014 & $1-0.2172768679$ & -0.0981725471 \\
\hline${ }^{1} g_{4}$ partial wave & & ${ }^{3} g_{4}$ partial wave & \\
\hline $0-0.0159359974$ & 0.0110169386 & $0-0.0762338541$ & 0.0498484441 \\
\hline $1-0.0229351778$ & 0.0073206473 & $1-0.1107702854$ & 0.0371277135 \\
\hline $2-0.0056121168$ & & $2-0.0295683403$ & \\
\hline
\end{tabular}

Table 2

Same as in Table 1 but for the coupled $N N$ waves.

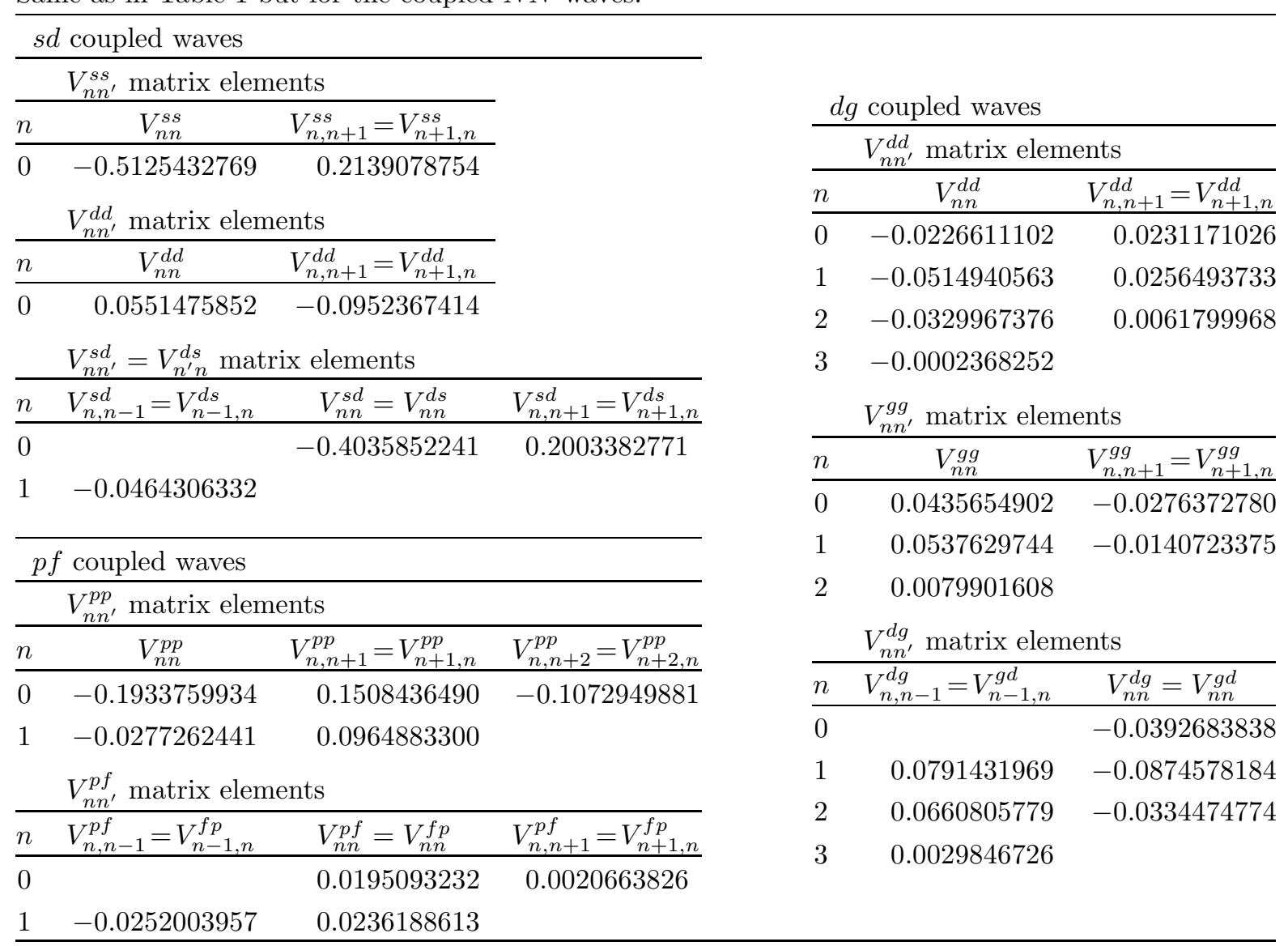




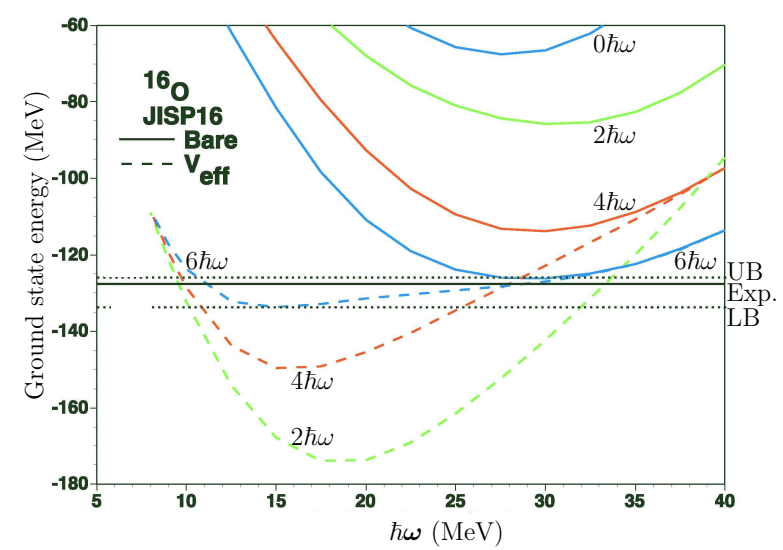

Figure 1. (Color online) The $\hbar \omega$ dependence of the ${ }^{16} \mathrm{O}$ ground state energy obtained with bare JISP16 and effective interaction based on JISP16 in a sequence of $N_{\max } \hbar \omega$ model spaces up to $N_{\max }=6$; the lines marked as Exp., UB and LB show the experimental ground state energy, the upper bound and the suggested lower bound for the NCSM ground state energy predictions.

pendence. This minimum is seen from Fig. 1 to ascend with increasing model space. Based on our results in lighter systems with larger spaces that show uniform convergence of this minimum, the minimum obtained in the $6 \hbar \omega$ model space is a suggested lower bound (LB) for the ground state energy. The difference between these upper and lower bounds is our estimate for the 'error bars' of our predictions. These error bars suggest reasonable convergence is attained but this requires verification in larger basis spaces.

Similar trends are found for most of the $p$ shell nuclei. We present in Table 3 their binding energies obtained with both bare and effective interactions. We also quote the $\hbar \omega$ values providing the minimum with the effective interaction. The difference between the given result and the result obtained with the same $\hbar \omega$ in the next smaller model space is presented in parenthesis to give an estimate of the convergence of our calculations. We quote our differences in significant figures from the rightmost figure of the stated result, omit-
Table 3

Binding energies (in $\mathrm{MeV}$ ) of nuclei obtained with bare JISP16 and effective interaction generated by JISP16.

\begin{tabular}{cccccc}
\hline Nucleus & Nature & Bare & Effective & $\begin{array}{c}\hbar \omega \\
(\mathrm{MeV})\end{array}$ & $\begin{array}{c}\text { ModeI } \\
\text { space }\end{array}$ \\
\hline${ }^{3} \mathrm{H}$ & 8.482 & 8.354 & $8.496(20)$ & 7 & $14 \hbar \omega$ \\
${ }^{3} \mathrm{He}$ & 7.718 & 7.648 & $7.797(17)$ & 7 & $14 \hbar \omega$ \\
${ }^{4} \mathrm{He}$ & 28.296 & 28.297 & $28.374(57)$ & 10 & $14 \hbar \omega$ \\
${ }^{6} \mathrm{He}$ & 29.269 & & $28.32(28)$ & 17.5 & $12 \hbar \omega$ \\
${ }^{6} \mathrm{Li}$ & 31.995 & & $31.00(31)$ & 17.5 & $12 \hbar \omega$ \\
${ }^{7} \mathrm{Li}$ & 39.245 & & $37.59(30)$ & 17.5 & $10 \hbar \omega$ \\
${ }^{7} \mathrm{Be}$ & 37.600 & & $35.91(29)$ & 17 & $10 \hbar \omega$ \\
${ }^{8} \mathrm{Be}$ & 56.500 & & $53.40(10)$ & 15 & $8 \hbar \omega$ \\
${ }^{9} \mathrm{Be}$ & 58.165 & 53.54 & $54.63(26)$ & 16 & $8 \hbar \omega$ \\
${ }^{9} \mathrm{~B}$ & 56.314 & 51.31 & $52.53(20)$ & 16 & $8 \hbar \omega$ \\
${ }^{10} \mathrm{Be}$ & 64.977 & 60.55 & $61.39(20)$ & 19 & $8 \hbar \omega$ \\
${ }^{10} \mathrm{~B}$ & 64.751 & 60.39 & $60.95(20)$ & 20 & $8 \hbar \omega$ \\
${ }^{10} \mathrm{C}$ & 60.321 & 55.26 & $56.36(67)$ & 17 & $8 \hbar \omega$ \\
${ }^{11} \mathrm{~B}$ & 76.205 & 69.2 & $73.0(31)$ & 17 & $6 \hbar \omega$ \\
${ }^{11} \mathrm{C}$ & 73.440 & 66.1 & $70.1(32)$ & 17 & $6 \hbar \omega$ \\
${ }^{12} \mathrm{~B}$ & 79.575 & 71.2 & $75.9(48)$ & 15 & $6 \hbar \omega$ \\
${ }^{12} \mathrm{C}$ & 92.162 & 87.4 & $91.0(49)$ & 17.5 & $6 \hbar \omega$ \\
${ }^{12} \mathrm{~N}$ & 74.041 & 64.5 & $70.2(48)$ & 15 & $6 \hbar \omega$ \\
${ }^{13} \mathrm{~B}$ & 84.453 & 73.5 & $82.1(67)$ & 15 & $6 \hbar \omega$ \\
${ }^{13} \mathrm{C}$ & 97.108 & 93.2 & $96.4(59)$ & 19 & $6 \hbar \omega$ \\
${ }^{13} \mathrm{~N}$ & 94.105 & 89.7 & $93.1(62)$ & 18 & $6 \hbar \omega$ \\
${ }^{13} \mathrm{O}$ & 75.558 & 63.0 & $72.9(62)$ & 14 & $6 \hbar \omega$ \\
${ }^{14} \mathrm{C}$ & 105.285 & 101.5 & $106.0(93)$ & 17.5 & $6 \hbar \omega$ \\
${ }^{15} \mathrm{O}$ & 104.659 & 103.8 & $106.8(77)$ & 20 & $6 \hbar \omega$ \\
\hline & 115.733 & 93.7 & $99.1(92)$ & 16 & $6 \hbar \omega$ \\
${ }^{15} \mathrm{O}$ & 111.956 & 110.1 & $115.8(126)$ & 16 & $6 \hbar \omega$ \\
& & & & & \\
& 114.4 & $119.5(126)$ & 16 & $6 \hbar \omega$ \\
\end{tabular}


Table 4

Ground state energy $E_{g s}$ and excitation energies $E_{x}$ (in $\mathrm{MeV}$ ), ground state point-proton rms radius $r_{p}$ (in $\mathrm{fm}$ ) and quadrupole moment $Q\left(\right.$ in $\left.e \cdot \mathrm{fm}^{2}\right)$ of the ${ }^{6} \mathrm{Li}$ nucleus; $\hbar \omega=17.5 \mathrm{MeV}$.

\begin{tabular}{|c|c|c|c|c|c|c|}
\hline $\begin{array}{c}\text { Interaction } \\
\text { Method } \\
\end{array}$ & Nature & $\begin{array}{c}\text { JISP6 } \\
\text { NCSM, } 10 \hbar \omega[6]\end{array}$ & $\begin{array}{c}\text { JISP16 } \\
\text { NCSM, } 12 \hbar \omega \\
\end{array}$ & $\begin{array}{c}\mathrm{AV} 8 '+\mathrm{TM} \\
\mathrm{NCSM}, 6 \hbar \omega[2]\end{array}$ & $\begin{array}{c}\text { AV18+UIX } \\
\text { GFMC [8 } 15]\end{array}$ & $\begin{array}{c}\text { AV18+IL2 } \\
\text { GFMC } 10 \quad 15\end{array}$ \\
\hline$E_{g s}\left(1_{1}^{+}, 0\right)$ & -31.995 & -31.48 & -31.00 & -31.04 & $-31.25(8)$ & $-32.0(1)$ \\
\hline$r_{p}$ & $2.32(3)$ & 2.083 & 2.151 & 2.054 & $2.46(2)$ & $2.39(1)$ \\
\hline$Q$ & $-0.082(2)$ & -0.194 & -0.0646 & -0.025 & $-0.33(18)$ & $-0.32(6)$ \\
\hline$E_{x}\left(3^{+}, 0\right)$ & 2.186 & 2.102 & 2.529 & 2.471 & $2.8(1)$ & 2.2 \\
\hline$E_{x}\left(0^{+}, 1\right)$ & 3.563 & 3.348 & 3.701 & 3.886 & $3.94(23)$ & 3.4 \\
\hline$E_{x}\left(2^{+}, 0\right)$ & 4.312 & 4.642 & 5.001 & 5.010 & $4.0(1)$ & 4.2 \\
\hline$E_{x}\left(2^{+}, 1\right)$ & 5.366 & 5.820 & 6.266 & 6.482 & & 5.5 \\
\hline$E_{x}\left(1_{2}^{+}, 0\right)$ & 5.65 & 6.86 & 6.573 & 7.621 & $5.1(1)$ & 5.6 \\
\hline
\end{tabular}

ting decimal points to save space. The ground state energy of $A=6,7$ and 8 nuclei converges uniformly from above with both the bare and effective interactions. We present in Table 3 only the effective interaction results for these nuclei due to their superior convergence features. For these nuclei, an extrapolation based on the fit by a constant plus exponential function for different $\hbar \omega$ values may be useful. For ${ }^{6} \mathrm{Li}$, this extrapolation results in a binding energy of 31.70(17) $\mathrm{MeV}$ where the value in parenthesis is the uncertainty of the fit. A similar extrapolation for ${ }^{6} \mathrm{He}$ results in a binding energy of $28.89(17) \mathrm{MeV}$ which is bound with respect to the $\alpha+n+n$ threshold. We note that the bare interaction results for $A=6$ nuclei are very close to the effective interaction ones demonstrating a remarkable softness of the JISP16 interaction: the ${ }^{6} \mathrm{Li}$ and ${ }^{6} \mathrm{He}$ binding energies are 30.94(44) and 28.23(41) $\mathrm{MeV}$ respectively, the extrapolations of the bare interaction bindings produce $31.33(12) \mathrm{MeV}$ for ${ }^{6} \mathrm{Li}$ and 28.61(12) MeV for ${ }^{6} \mathrm{He}$.

The nuclear Hamiltonian based on the ab exitu realistic $N N$ interaction JISP16, is seen to reproduce well the binding energies of nuclei with $A \leq 16$. The lowest state of natural parity has the correct total angular momentum in each nucleus studied. The experimental binding energies of all nuclei presented in Table 3 either lie within error bars of our predictions or are close to our sug- gested LB based on the effective interaction calculations. Generally JISP16 slightly underbinds only nuclei in the middle of the $p$ shell. The difference between UB and LB is small, suggesting that JISP16 provides good convergence. However, our error bars increase as binding energy decreases in a chain of isobars (cf the results for ${ }^{13} \mathrm{O}$ and ${ }^{13} \mathrm{~N}$ ).

We present in Tables 4 and 5 spectra and ground state properties of ${ }^{6} \mathrm{Li}$ and ${ }^{10} \mathrm{~B}$ which are known 28 8[15]16] to be sensitive to an explicit $N N N$ interaction. Here, the $a b$ exitu JISP16 $N N$ interaction alone provides a good description. The JISP $16{ }^{6} \mathrm{Li}$ spectrum seems to be less favorable than that provided by our JISP 6 interaction specifically fitted to the ${ }^{6} \mathrm{Li}$ spectrum. However, the JISP $16{ }^{6} \mathrm{Li}$ spectrum is competitive with those of realistic $N N+N N N$ potential models. Also, we obtain a good description of the ${ }^{6} \mathrm{Li}$ quadrupole moment $Q$ that is a recognized challenge due to a delicate cancellation between deuteron quadrupole moment and the $d$ wave component of the $\alpha-d$ relative wave function. We observe that $Q$ and the point-proton rms radius $r_{p}$ have a more prominent $\hbar \omega$ dependence than the binding energy.

The ${ }^{10} \mathrm{~B}$ properties are also seen to be welldescribed with the JISP16 interaction contrary to previous results from pure realistic $N N$ interactions [2]16. We note that the ${ }^{10} \mathrm{~B}$ spectrum depends on $\hbar \omega$ at $N_{\max }=8$ but not so strongly 
Table 5

Same as in Table 4 but for the ${ }^{10} \mathrm{~B}$ nucleus; $\hbar \omega=15 \mathrm{MeV}$.

\begin{tabular}{|c|c|c|c|c|}
\hline $\begin{array}{c}\text { Interaction } \\
\text { Method } \\
\end{array}$ & Nature & 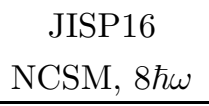 & $\begin{array}{c}\text { AV8'+TM' } \\
\text { NCSM, } 4 \hbar \omega[2]\end{array}$ & $\begin{array}{l}\text { AV18+IL2 } \\
\text { GFMC [16] }\end{array}$ \\
\hline$E_{g s}\left(3_{1}^{+}, 0\right)$ & -64.751 & -60.14 & -60.57 & $-65.6(5)$ \\
\hline$r_{p}$ & $2.30(12)$ & 2.168 & 2.168 & $2.33(1)$ \\
\hline$Q$ & $+8.472(56)$ & 6.484 & +5.682 & $+9.5(2)$ \\
\hline$E_{x}\left(1_{1}^{+}, 0\right)$ & 0.718 & 0.555 & 0.340 & 0.9 \\
\hline$E_{x}\left(0^{+}, 1\right)$ & 1.740 & 1.202 & 1.259 & \\
\hline$E_{x}\left(1_{2}^{+}, 0\right)$ & 2.154 & 2.379 & 1.216 & \\
\hline$E_{x}\left(2_{1}^{+}, 0\right)$ & 3.587 & 3.721 & 2.775 & 3.9 \\
\hline$E_{x}\left(3_{2}^{+}, 0\right)$ & 4.774 & 6.162 & 5.971 & \\
\hline$E_{x}\left(2_{1}^{+}, 1\right)$ & 5.164 & 5.049 & 5.182 & \\
\hline$E_{x}\left(2_{2}^{+}, 0\right)$ & 5.92 & 5.548 & 3.987 & \\
\hline$E_{x}\left(4^{+}, 0\right)$ & 6.025 & 5.775 & 5.229 & 5.6 \\
\hline$E_{x}\left(2_{2}^{+}, 1\right)$ & 7.478 & 7.776 & 7.491 & \\
\hline
\end{tabular}

as to alter our main conclusions. For example, the minimum of the ${ }^{10} \mathrm{~B}$ ground state corresponds to $\hbar \omega=20 \mathrm{MeV}$ while the minimum in the first excited state energy occurs at $\hbar \omega=15 \mathrm{MeV}$. We present in Table 5 the ${ }^{10} \mathrm{~B}$ properties obtained with $\hbar \omega=15 \mathrm{MeV}$, i.e. with the $\hbar \omega$ value corresponding to the minimum of the first excited state since it has a more pronounced $\hbar \omega$ dependence than the ground state. The ${ }^{10} \mathrm{~B}$ ground state spin was not previously reproduced with a pure realistic $N N$ interaction. We observe that our description of the ${ }^{10} \mathrm{~B}$ spectrum is somewhat better than the one obtained with the Argonne AV8' $N N$ potential and Tucson-Melbourne TM' $N N N$ force. In particular, we reproduce the ordering of ${ }^{10} \mathrm{~B}$ levels except for the $\left(3_{2}^{+}, 0\right)$ state. We note that the $\left(3_{2}^{+}, 0\right)$ state is also too high with the AV8' + TM' interaction.

In constructing ISTP [5, JISP6 6] and JISP16 potentials we adopted only the accepted symmetries of the $N N$ interaction and neglected explicit constraints such as the long-range behavior from meson-exchange theory. However, this does not mean that the JISP16 NN interaction is inconsistent with meson-theoretical forms of the $N N$ interaction. On the contrary, it is well-known that the one-pion exchange (OPE) dominates the $N N$ interaction in higher partial waves and the longrange behavior of $N N$ interaction in lower partial waves. In this context, we showed in Ref. [5] that our scattering wave functions in higher partial waves are nearly indistinguishable from those of the Nijmegen-II OPE potential. Also, in lower partial waves, our wave functions are very close to those of Nijmegen-II at large distances and a small difference is seen only at higher energies. Finally, we introduced the PETs of JISP6 and JISP16 only in lower partial waves and only in a few lowest oscillator components of the potential with a large value of $\hbar \omega=40 \mathrm{MeV}$. As a result, PETs reshape the wave functions at short distances $(\lesssim 1 \mathrm{fm})$. Thus, the JISP16 interaction appears to be consistent with the well-established OPE tail as embodied in the Nijmegen-II $N N$ interaction.

We propose our ab exitu JISP16 as a realistic $N N$ interaction since it describes the two-body observables with high precision. In addition, it provides a reasonable and economic description of properties of many-body nuclear systems in the microscopic NCSM approach. Economy arises from the softness of the interaction represented 
in a separable oscillator form. Short distance phase-equivalent transformations adjust the offshell properties successfully to reduce the roles of multi-nucleon interactions. The particular mechanism of this reduction is not clear at the present time. However, our results as well as the success of the approach of Ref. [17, clearly demonstrate that such a mechanism exists and should be studied in detail. We plan to study this with explicit $N N N$ interactions.

We conclude that the many-body nuclear Hamiltonian obtained in our $a b$ exitu approach is realistic from the point of view of providing a good description of a wide range of nuclear data. The suggested JISP16 $N N$ interaction opens a path for extending realistic microscopic theory to heavier nuclei, to achieve better convergence and to obtain improved agreement with experiment.

This work was supported in part by the Russian Foundation of Basic Research grant No 05-02-17429, by US NSF grant No PHY-0071027 and under the auspices of the US Department of Energy by the University of California, Lawrence Livermore National Laboratory under contract No. W-7405-Eng-48 and under US DOE grants DE-FG-02 87ER40371 and DEAC02-76SF00515.

\section{REFERENCES}

1. P. Navrátil, J. P. Vary, B. R. Barrett, Phys. Rev. Lett. 84, 5728 (2000); Phys. Rev. C 62, 054311 (2000).

2. P. Navrátil, W. E. Ormand, Phys. Rev. C 68, 034305 (2003).

3. A. Nogga, P. Navrátil, B. R. Barrett, J. P. Vary, Phys. Rev. C 73, 064002 (2006).

4. S. A. Zaitsev, Theor. Math. Phys. 115, 575 (1998).

5. A. M. Shirokov, A. I. Mazur, S. A. Zaytsev, J. P. Vary, T. A. Weber, Phys. Rev. C 70, 044005 (2004).

6. A. M. Shirokov, J. P. Vary, A. I. Mazur, S. A. Zaytsev, T. A. Weber, Phys. Lett. B 621, 96 (2005).

7. H. Kamada, A. Nogga, W. Glöckle, E. Hiyama, M. Kamimura, K. Varga, Y. Suzuki, M. Viviani, A. Kievsky, S. Rosati,
J. Carlson, S. C. Pieper, R. B. Wiringa, P. Navrátil, B. R. Barrett, N. Barnea, W. Leidemann, G. Orlandini, Phys. Rev. C 64, 044001 (2001).

8. B. S. Pudliner, V. R. Pandharipande, J. Carlson, S. C. Pieper, R. B. Wiringa, Phys. Rev. C 56, 1720 (1997).

9. R. B. Wiringa, Nucl. Phys. A 631, 70c (1998).

10. S. C. Pieper, V. R. Pandharipande, R. B. Wiringa, J. Carlson, Phys. Rev. C 64, 014001 (2001).

11. R. B. Wiringa, R. A. Smith, T. L. Ainsworth, Phys. Rev. C 29, 1207 (1984);

R. B. Wiringa, V. G. J. Stoks, R. Schiavilla, Phys. Rev. C 51, 38 (1995).

12. J. Morales, Jr., V. R. Pandharipande, D. G. Ravenhall, Phys. Rev. C 66, 054308 (2002).

13. W. N. Polyzou, W. Glöckle, Few-Body Syst. 9, 97 (1990).

14. R. Machleidt, private communication.

15. S. C. Pieper, R. B. Wiringa, J. Carlson, Phys. Rev. C 70, 054325 (2004).

16. S. C. Pieper, K. Varga, R. B. Wiringa, Phys. Rev. C 66, 044310 (2002).

17. P. Doleschall and I. Borbély, Phys. Rev. C 62, 054004 (2000). 